\title{
Optimization of Zoom Lens with Discrete State of Liquid Lens Elements by Using Genetic Algorithm
}

\author{
Cheng-Mu Tsai \\ Graduate Institute of Precision Engineering, National Chung Hsing University, Taichung 402, Taiwan \\ Correspondence should be addressed to Cheng-Mu Tsai; jmutsai@gmail.com
}

Received 20 September 2014; Revised 7 December 2014; Accepted 7 December 2014

Academic Editor: Giuseppe Carbone

Copyright ( $) 2015$ Cheng-Mu Tsai. This is an open access article distributed under the Creative Commons Attribution License, which permits unrestricted use, distribution, and reproduction in any medium, provided the original work is properly cited.

\begin{abstract}
This paper is to employ liquid lens elements to design a lens with zoom function by using the genetic algorithm (GA) optimization. The liquid lens elements used in the proposal can apply voltage adjustment to generate the electrical field that induces the liquid with electric conductivity to vary the surface curvature between two different kinds of liquids. According to the voltage level, the liquid lens element makes the discrete variation of the curvature and thickness realize the zoom function without moving the lens groups so that the overall length can be reduced. However, it is difficult to design the zoom lens under the discrete variation of the curvature and thickness in the liquid lens elements and the mechanical space that is constantly limited. The GA offers a flexible way for lens optimization. We regarded the spot size as the fitness function to look for the optimum curvatures, thickness, and the corresponding statuses of liquid lens elements for the zoom lens. As a result, the zoom lens with constant space can be realized by running the selection, crossover, and mutation operation in the GA optimization.
\end{abstract}

\section{Introduction}

Mobile devices are very popular today. The camera function has been integrated as a basic demand in current mobile devices for taking an object's picture with constant distance. When an object needs to be enlarged or shrunk for different distances, the only one way the user can perform this is to take the lens close to or far away from the object. In some cases, the user cannot move close to the object, which results in that the image of the distant object cannot be enlarged at all. Thus, developing a lens with zoom function is attracting in the mobile device. Traditional zoom lens designs are based on smoothly moving lens groups by using analog mechanical camera control, which requires larger room space to adjust the position of the lens groups. Current digital camera applies this kind of method to employ three to six lens elements for zoom function implement.

However, mobile devices are usually required in a thin thickness. No more space is available for the lens elements moving for the zoom function implement. Therefore, it is a big challenge for designing a lens with zoom function in the mobile device, especially for no moving lens elements. Since the zoom lenses are to adjust their effective focal length (EFL) for various image sizes, some lens elements are developed to change their EFLs to design the zoom lens without lens elements moving. One of focus variation lens elements, called liquid crystal lens (LC lens) $[1,2]$, has been developed to realize a zoom lens. This kind of lens element is to align the LC cells to compose the refractive index that is like graded index. Modifying the LC cells alignment would change the lens element optical power. However, the LC lens cannot allow the high optical flux to expose on the image plane because the light needs to be polarization-dependent in the LC cells.

Another focus variation lens element is the liquid lens element [3-7]. Many kinds of technologies were developed to implement the liquid lens elements such as using pressureinduced [4], thermal effect [5], dielectrophoresis [6], and electrowetting [7]. The liquid lens in [4] is to use two substrates to seal a thin elastic membrane on the outer surface of top aperture and seal on the inner surface of the bottom aperture. When one applies pressure to the outer membrane, the liquid would redistribute inside to result in swelling of the inner membrane for forming a planoconvex lens. The liquid lens in [5] applies the thermal effect on the liquid 


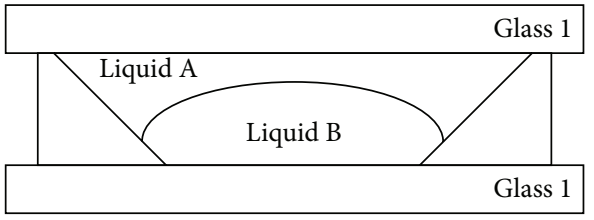

(a)

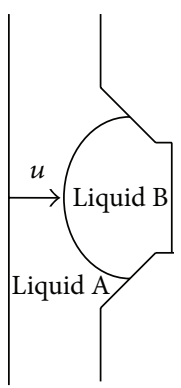

(b)

FIgURE 1: Liquid lens [19] (a) structure; (b) cross-section of a liquid lens.

with thermal sensitivity to change the surface curvature. With the dielectrophoretic effect [6], the generated dielectric force pushes the liquid into its neighboring hole to make the curvature difference by tuning a voltage. The liquid lens by using electrowetting [7] uses two different kinds of liquids. One liquid is conductive and the other is nonconductive. When one tunes the voltage to change the electrical field, the liquid with conductive property would be induced to change the surface curvature between two different kinds of liquids. The electrowetting liquid lens can support the EFL from $5 \mathrm{~mm}$ to infinity. Its tuning duration can be limited within $5 \mathrm{~ms}$. We apply this kind of liquid lens to design a zoom lens for mobile device.

There are many applications by using electrowetting liquid lens [8-10]. Two different kinds of structures with liquid lens elements have been presented to miniature the lens for laparoscopy [8]. Since the optical power of liquid lens is not large enough, Fang et al. [9] employed two liquid lenses with moving some lens elements to realize a zoom lens for nine segment focuses. However, the goal of using liquid lens is to realize a zoom lens without moving any lens elements. In order to increase the optical power, the proposal employs four liquid lenses. These liquid lenses are used to change the image zoom as well as compensating the image quality. Based on the Cooke Triple Lens, we modify the structure with four liquid lens elements to implement a zoom lens without moving any lens elements. However, it is very difficult to find out the appropriate parameters such as curvature and thickness for a lens, especially for zoom lens design with discrete parameters. Although the common commercial optical software provides some optimum methods like damping least square (DLS) $[11,12]$ to find out the great parameters as much as possible, the optimized process requires the domain of function to be continuous rather than the discrete state. Recently, another great optimum method with global optimization called genetic algorithm (GA) is applied for some applications [13-18], which can find the solution with discrete state.

The proposal only employed the liquid elements to achieve the zoom function without moving any lens group. There are 29 statuses of each liquid element to be referred to design the zoom lens. The designer is unable to find the optimal statuses of liquid elements by using the optimal function (including the built-in GA) of commercial optical software. In this paper, we regard the status of liquid element
TABLE 1: The refractive index of Glass 1 and Glass 2 [19].

\begin{tabular}{ll}
\hline$(\mu \mathrm{m})$ & Index \\
\hline 700.0 & 1.5150 \\
650.0 & 1.5164 \\
590.0 & 1.5187 \\
550.0 & 1.5206 \\
480.0 & 1.5252 \\
430.0 & 1.5300 \\
\hline
\end{tabular}

TABLE 2: The refractive index of liquid lens [19].

\begin{tabular}{lcc}
\hline Wavelength & $\begin{array}{c}\text { PC100 } \\
\text { (liquid A) }\end{array}$ & $\begin{array}{c}\text { H100 } \\
\text { (liquid B) }\end{array}$ \\
\hline 400 & 1.41178 & 1.51297 \\
448 & 1.40729 & 1.50340 \\
489 & 1.40451 & 1.49772 \\
541 & 1.40180 & 1.49250 \\
589.3 & 1.39988 & 1.48894 \\
654.6 & 1.39791 & 1.48535 \\
703 & 1.39671 & 1.48332 \\
\hline
\end{tabular}

as the gens of the GA to look for the optimal status. The GA was written in the macro to find the best status and other optimal parameters of the proposed lens. By running the functions selection, crossover, and mutation, the GA is used to optimize the zoom lens for using in mobile device. As expected, a lens with $1.4 \mathrm{x}$ zoom ratio can be implemented without moving any lens elements. The organization of this paper is as follows. In Section 2 an initial layout is built by using the commercial software. Section 3 represents the process of GA and its role of optimization in lens design. The paper shows the simulation results and discusses them in Section 4. Conclusions are drawn in Section 5.

\section{Initial Layout}

The liquid lens element used in the proposal is the ARCTIC 416 [19] that is electrowetting liquid lens, as shown in Figure 1(a). It consists of two different kinds of liquids and two solid glasses. The refractive indices of the glass materials are shown in Table 1. Table 2 shows the refractive indices of two 


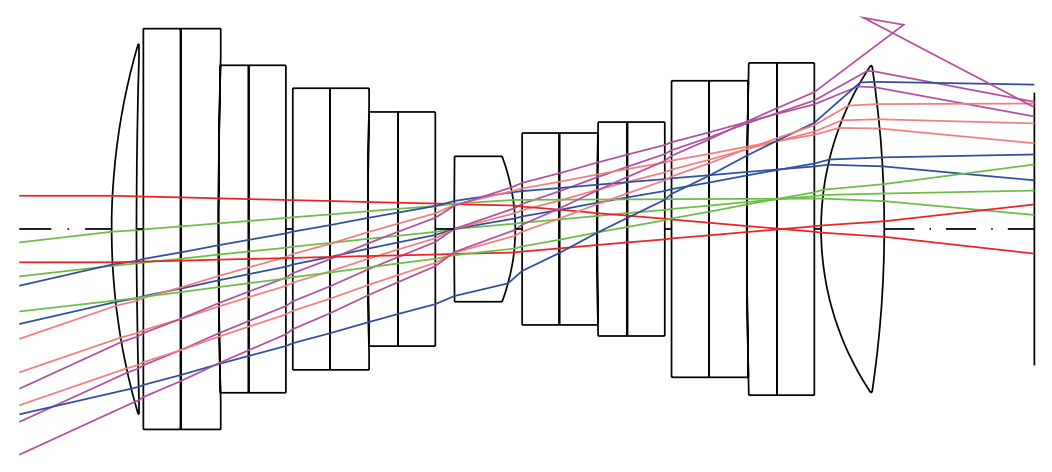

FIGURE 2: Initial lens layout.

kinds of liquids for various wavelengths. When the voltage is adjusted to induce the electric filed for one kind of liquids, the surface curvature between the two liquids would be changed in the liquid lens element. Table 3 lists the relation between the surface curvature of the two liquids and the voltage values. For example, when the voltage is tuned to 33 volt in the liquid lens element, the curvature radius between two liquids shown in Figure 1(b) is $-21.242 \mathrm{~mm}$. The corresponding thickness is $0.709 \mathrm{~mm}$ in liquid A. Because the overall length of liquid A and liquid B is $1 \mathrm{~mm}$, the thickness in liquid B is $0.291 \mathrm{~mm}$.

Most lenses in mobile device are designed by applying the Cooke triplet structure for miniaturizing the space. Based on the same structure, the liquid lens elements are inserted among lens elements to realize the zoom lens through varying the curvature between two kinds of liquids. Two liquid lens groups are employed to implement the functions focus and image quality compensating. However, the refractive index difference between two liquids is small, which results in less optical power that can be manipulated to facilitate the design of zoom function. By placing additional lens elements, the requirement of optical power can be alleviated in using electrowetting liquid lens. Two liquid lens groups are, respectively, placed into the two spaces between lens elements of Cooke triplet structure, as shown in Figure 2. The initial layout is regarded as the structure that runs the optimization for finding out the best parameters. The common commercial optical software is to apply the DLS to optimize the lens system. However, the optimized result almost depends on the initial parameters so that the results easily fall into the local optimization. Moreover, we must follow the specification of liquid lens element shown in Table 3. It is difficult to optimize the image quality according to the data of Table 3. Instead, we create a macro with the GA optimization to design the zoom lens with liquid lens elements.

\section{Zoom Lens Design with Liquid Lens Based on Genetic Algorithm}

In the optical system design, the designer is responsible for searching the parameters that make the outcome meet the specification requirement. This is similar to the creatures that have to explore some ways to accommodate their living circumstance; that is, the creature/lens would accommodate
TABLE 3: The relation between the surface curvature, thickness, and driving voltage [19].

\begin{tabular}{lcc}
\hline Voltage $(\mathrm{V})$ & $R(\mathrm{~mm})$ & $u(\mathrm{~mm})$ \\
\hline 0 to 32 & -17.896 & 0.720 \\
33 & -21.242 & 0.709 \\
34 & -26.402 & 0.699 \\
35 & -35.082 & 0.688 \\
36 & -52.731 & 0.678 \\
37 & -108.02 & 0.667 \\
38 & 1635.997 & 0.656 \\
39 & 93.995 & 0.645 \\
40 & 48.026 & 0.635 \\
41 & 32.095 & 0.624 \\
42 & 24.014 & 0.613 \\
43 & 19.13 & 0.602 \\
44 & 15.862 & 0.59 \\
45 & 13.521 & 0.579 \\
46 & 11.764 & 0.568 \\
47 & 10.396 & 0.557 \\
48 & 9.302 & 0.545 \\
49 & 8.408 & 0.534 \\
50 & 7.663 & 0.522 \\
51 & 7.033 & 0.511 \\
52 & 6.494 & 0.499 \\
53 & 6.028 & 0.487 \\
54 & 5.62 & 0.475 \\
55 & 5.262 & 0.463 \\
56 & 4.943 & 0.451 \\
57 & 4.659 & 0.439 \\
58 & 4.404 & 0.427 \\
59 & 4.174 & 0.415 \\
60 & 3.965 & 0.402 \\
\hline & &
\end{tabular}

to/meet the growing circumstance/specification requirement as much as possible. The specification of lens in the GA is equivalent to the accommodating living circumstance for the creatures. In general, there are many individuals of the same kind of creature in the environment. The population size is usually related to the accommodating 
evolution generation. Let the population size be pop_size for the same kind of creature/lens. The pop_size same kind of creatures/lenses would compete with one another. The better one would gain more probability to propagate its great genes into the next generation offspring. By using a GA object function, the competences of all individuals of the same kind of creatures/lenses are evaluated to judge the ability of propagation. The value calculated from the object function is usually toward zero to represent the lens approaching all the specification requirements. Our target is to search the parameters that are satisfied for a zoom lens with liquid lens elements. The object function involves the values about the multiple EFLs and image quality; that is,

$$
\begin{aligned}
& \operatorname{obj} \text { Value }(i)=\sum_{z=1}^{2} w_{z f} \max \left\{\left|\mathrm{SPO}_{f}^{z}\right|\right\} \\
& +\left\{\begin{array}{cc}
0 & \text { for }\left|\mathrm{EFL}_{\mathrm{GA}}^{z}-\mathrm{EFL}_{S}^{z}\right|<0.15 \\
& \sum_{z=1}^{2}\left[w_{z}\left|\mathrm{EFL}_{\mathrm{GA}}^{z}-\mathrm{EFL}_{S}^{z}\right|\right]
\end{array}\right. \\
& \text { for } i=1,2, \ldots, \text { pop_size, }
\end{aligned}
$$

where $z$ is the number of zoom segments. The $\mathrm{EFL}_{S}^{z}$ is the EFL for the specification, that is, $5 \mathrm{~mm}$ and $7 \mathrm{~mm}$. The $\mathrm{EFL}_{\mathrm{GA}}^{z}$ is the EFL value evaluated from the GA lens individual. The $\mathrm{SPO}_{f}^{z}$ is root mean square (rms) spot diameter at the field $f$ and the zoom segment $z \cdot w_{z}$ and $w_{z f}$ are the weighting, whose defaults are 1 . The weighting can be increased to emphasize some terms in (1). The weightings $w_{z}$ and $w_{z f}$ in the proposal are 100 and 1 , respectively.

The curvatures and thickness on each lens element are regarded as the genes used to optimize the zoom lens. These genes are randomly produced to build a lens structure when starting running GA. There are pop_size (population size) lens structures randomly built by using the same procedure in the first generation. The optical software is applied to calculate the spot diameter and EFL for all the lens structures. According to (1), the object value is obtained to evaluate how fit the lens structure meets specification requirement (i.e., meet EFLs $5 \mathrm{~mm}$ and $7 \mathrm{~mm}$ and small spot diameter). These object values from all the lens structures are used to perform the operation crossover. We need to select at least two groups of genes of lens structure to run the operation crossover. Among the selection methods, the roulette wheel [13] is widely applied to determine which lens structures would be as the parents when running the crossover. It is to allocate the area of roulette wheel based on how fit the lens structure meets specification requirement. The more fit degree the lens structure is, the more area of roulette wheel the lens structure is. Each lens structure is assigned a piece of area in the roulette wheel according to its fitness. We randomly assign a point in the roulette wheel whose area of location corresponds to one of lens structures. The corresponding lens structure is determined to be one of the parents. The same procedure is also used to determine the other parent to implement operation crossover. However, the definition of object value is inversely scalar to the size of the piece of area in the roulette wheel. Therefore, an appropriate function called fitness function is transformed from the object function to evaluate the fit degree; that is,

$$
\begin{aligned}
\text { fit_Value }(i)= & \max \left\{o b j \_ \text {Value }(i)\right\} \\
& +\min \left\{o b j \_ \text {Value }(i)\right\}-\text { obj_Value }(i) \\
& \text { for } i=1,2, \ldots, \text { pop_size },
\end{aligned}
$$

where the $\max \{\}$ and $\min \{\}$ are the functions about the maximum and minimum object values among the pop_size lens structures, respectively. The roulette wheel uses the fitness values and probability concept to allocate the area of each lens individual. The probability area of each lens individual in roulette wheel is defined as

$$
p(i)=\frac{\text { fit_Value }(i)}{\sum_{n=1}^{\text {pop_size }} \text { fit_Value (n) }} \text { for } i=1,2, \ldots, \text { pop_size. }
$$

According to (3), the lens individual can obtain its area probability. The summation area probability of all lens individuals is 1 . The cumulative distribution probability (CDP) on each lens individual can be derived from

$$
\begin{gathered}
q(i)=\frac{\sum_{n=1}^{i} p(n)}{\sum_{m=1}^{\text {pop_size }} p(m)} \quad \text { for } i=1,2, \ldots, \text { pop_size, } \\
q(0)=0 .
\end{gathered}
$$

Using the CDP on (4a) and (4b), a random number $\alpha$ ranged from 0 to 1 is generated to perform the operation selection. The $i$ th lens individual is regarded as one of the parents of the crossover operation if $q(i-1)<\alpha \leqq q(i)$. The same procedure is also applied to the other parent to run the selection. Let two parents be gene sequences $X=\left(x_{1}, x_{2}, \ldots, x_{N}\right)$ and $Y=\left(y_{1}, y_{2}, \ldots, y_{N}\right)$. Their offspring is presented as $Z=\left(z_{1}, z_{2}, \ldots, z_{N}\right)$. There are many ways to implement the operation crossover. Which method is used depends on the property of gene parameters. The curvature of liquid lens between two liquids is according to Table 3 that shows 29 statuses. The gene for liquid lens element would be one of 29 statuses. We use the random crossover to determine which status is propagated to the next generation. The random crossover first is to choose a number between 0 and 1 . This number is often set as 0.5 . The next step is to produce another random number $\beta$ ranged from 0 to 1 . Two genes from two different parents are exchanged if $\beta>0.5$. Figure 3 shows the random numbers $\beta$ on genes 2,3 , and $N-1$ are larger than 0.5 so that these genes are exchanged from two parents.

The offspring gene for the liquid element status can be determined by using the random crossover. However, the other genes such as curvature and thickness are not suitable for using the random crossover because these genes would be mixed from the two parents. Thus, another crossover operation is defined for the curvature and thickness; that is,

$$
z_{i}=\beta x_{i}+(1-\beta) y_{i},
$$




\begin{tabular}{|c|c|c|c|c|c|}
\hline$\beta=0.3$ & $\beta=0.7$ & $\beta=0.6$ & $\cdots$ & $\beta=0.8$ & $\beta=0.1$ \\
\hline$x_{1}$ & $x_{2}$ & $x_{3}$ & $\cdots$ & $x_{N-1}$ & $x_{N}$ \\
\hline$y_{1}$ & $y_{2}$ & $y_{3}$ & $\cdots$ & $y_{N-1}$ & $y_{N}$ \\
\hline \multicolumn{6}{|c|}{$\begin{array}{l}\text { After } \\
\text { crossover }\end{array}$} \\
\hline$\beta=0.3$ & $\beta=0.7$ & $\beta=0.6$ & $\ldots$ & $\beta=0.8$ & $\beta=0.1$ \\
\hline$x_{1}$ & $y_{2}$ & $y_{3}$ & $\cdots$ & $y_{N-1}$ & $x_{N}$ \\
\hline$y_{1}$ & $x_{2}$ & $x_{3}$ & $\cdots$ & $x_{N-1}$ & $y_{N}$ \\
\hline
\end{tabular}

FIgURE 3: A scheme of random crossover.

where $\beta$ is a random number between 0 and 1 . The proposal applies the random crossover for determining the status of liquid lens element and uses (5) for deriving the curvature and thickness of standard lens element. The operations selection and crossover provide a good way to propagate the better genes into next generation. However, the creatures are probably to explore their best for living in the nature environment. During the evolution process, not only the better genes propagate to next generation but also the creatures might try to modify their genes to meet the environment circumstance more. Another way to obtain the better genes is to employ the mutation process. We define a mutation rate, $p_{m}$, that is a number between 0 and 1 . A random number $\alpha$ is generated from 0 to 1 . The mutation operation is implemented for the corresponding genes $z_{i}^{\prime}$ when the random number $\alpha$ is less than $p_{m}$. There are two kinds of mutation process used in the proposal. One is used to randomly select the curvature status of liquid lens element. The mutation for such discrete process is defined as

$$
z_{i}^{\prime}=z_{i} \pm \bmod (\text { random integer number, } \Delta+1),
$$

where $\Delta$ is a constrained value to avoid divergence. The $\bmod ()$ is a module function; for example, $\bmod (5,3)=2$. The other mutation process for the curvature and thickness is defined as

$$
z_{i}^{\prime}=z_{i} \pm \beta \Delta,
$$

where $\Delta$ is also a constrained value. The $\beta$ is a random number between 0 and 1 . Using the selection, crossover, and mutation, the GA is written as macro in an optical software to optimize a zoom lens with liquid lens elements. Figure 4 shows the flow chart of GA.

\section{Simulation Results and Discussions}

During the GA optimization, although we can obtain the better genes based on the larger population size, another way to gain the better genes is the evolution with high mutation rate. It is probably difficult, however, to converge in lower object value for high mutation rate. The suggestion is to use larger population size for the GA optimization. Although it must spend longer time, the final result is usually better than that of the optimization with high mutation rate. In general,

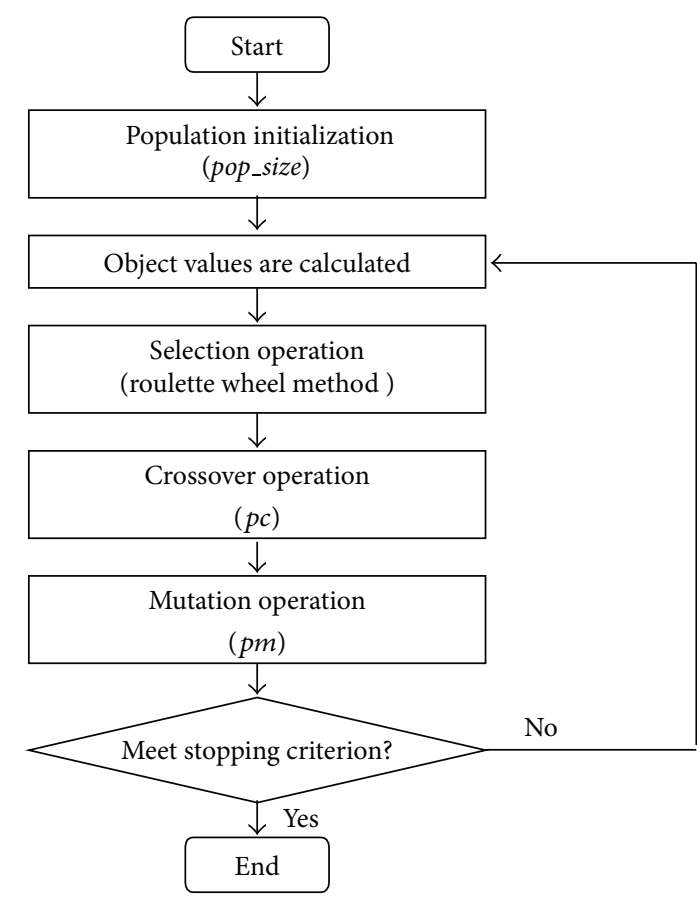

FIgURE 4: The GA flow chart.

the lenses from the initial genes are unable to meet the specification requirement so that the contemporary parent lenses have to reproduce the offspring lenses. Two parent lenses are selected from the roulette wheel method to mix their genes with curvature and thickness for running the operation crossover.

After the crossover, the operation mutation offers an opportunity to modify the genes values out of that of their parents. In general, the mutation rate is set lower than 0.2 with large population size. The operations selection, crossover, and mutation are applied to create the offspring lenses. The crossover rate is set to 0.8 in this paper; that is, when the population size is 2000 , the amount of the offspring is 1600 . The best $20 \%$ of individual lenses are kept to the next generation because they have better genes to support their longer life than others. The contemporary population size still keeps 2000 for the next generation. All operations are running until meeting the specification.

Four liquid lens elements were applied to design a zoom lens used in mobile devices. According to Table 3, the thicknesses of two liquids and the surface curvature between two kinds of liquids are related to the voltage. The proposed lens design must satisfy one of 29 discrete statuses of liquid element. As the curvature changed, the thicknesses of both liquids must also be modified to make the optimization more difficult for commercial software because it does not support the discrete optimization. Therefore, these three parameters cannot be set as variables when running optimization in the common commercial optical software. Instead, the GA was used to optimize the lens design. The thicknesses of Glass 1 and Glass 2 shown in Figure 1(a) and the other parameters in the three lens elements were also optimized by using the GA. However, the proposal was to modify the liquid lens 
TABLE 4: The factors after the GA optimization.

\begin{tabular}{|c|c|c|c|c|}
\hline Surface number & Surface type & $Y$ curvature & Thickness & Glass \\
\hline Object & Sphere & 0.0000 & Infinity & \\
\hline 1 & Sphere & 0.0448 & 3.5000 & 487490.704058 \\
\hline 2 & Sphere & 0.0926 & 0.5883 & \\
\hline 3 & Sphere & 0.0000 & 0.7837 & "Glass 1" \\
\hline 4 & Sphere & 0.0000 & $0.624 / 0.439$ & "PC100" \\
\hline 5 & Sphere & $0.0312 / 0.2146$ & $0.376 / 0.561$ & “H100" \\
\hline 6 & Sphere & 0.0000 & 0.4238 & “Glass 1" \\
\hline 7 & Sphere & 0.0000 & 0.1000 & \\
\hline 8 & Sphere & 0.0000 & 0.5892 & “Glass 1" \\
\hline 9 & Sphere & 0.0000 & $0.667 / 0.511$ & "PC100" \\
\hline 10 & Sphere & $-0.0093 / 0.1422$ & $0.333 / 0.489$ & “H100" \\
\hline 11 & Sphere & 0.0000 & 0.3038 & "Glass 1" \\
\hline 12 & Sphere & 0.0000 & 5.0515 & \\
\hline Stop & Sphere & -0.0051 & 3.1314 & 487490.704058 \\
\hline 14 & Sphere & -0.2924 & 0.2946 & \\
\hline 15 & Sphere & 0.0000 & 0.5753 & “Glass 1" \\
\hline 16 & Sphere & 0.0000 & $0.402 / 0.720$ & "PC100" \\
\hline 17 & Sphere & $0.2522 /-0.0559$ & $0.598 / 0.280$ & “H100” \\
\hline 18 & Sphere & 0.0000 & 0.5397 & "Glass 1" \\
\hline 19 & Sphere & 0.0000 & 0.1 & \\
\hline 20 & Sphere & 0.0000 & 0.5322 & "Glass 1" \\
\hline 21 & Sphere & 0.0000 & $0.415 / 0.667$ & “PC100" \\
\hline 22 & Sphere & $0.2396 /-0.0093$ & $0.585 / 0.333$ & “H100" \\
\hline 23 & Sphere & 0.0000 & 0.6313 & "Glass 1" \\
\hline 24 & Sphere & 0.0000 & 0.4923 & \\
\hline 25 & Sphere & 0.1873 & 2.7430 & 743972.448504 \\
\hline 26 & Aspherical & 0.6168 & 0.4385 & \\
\hline Image & Sphere & 0.0000 & -0.0002 & \\
\hline
\end{tabular}

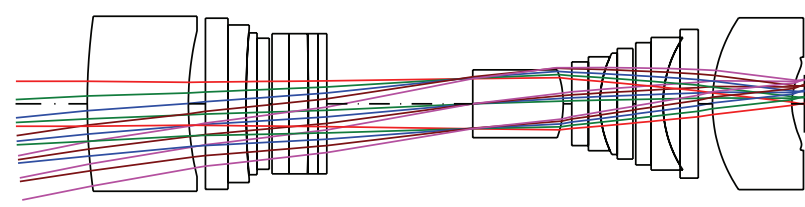

(a)

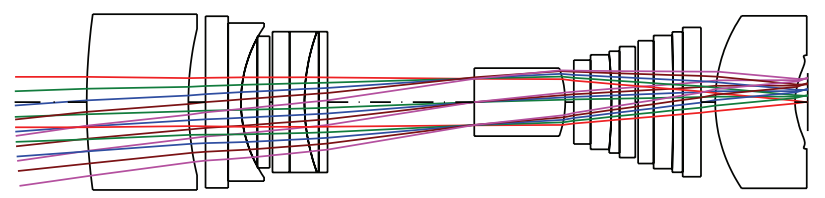

(b)

FIGURE 5: 1.4x zoom lens with liquid lens elements. (a) $\mathrm{EFL}=5 \mathrm{~mm}$ and (b) $\mathrm{EFL}=7 \mathrm{~mm}$.

elements for implementing the zoom function. Table 3 shows one liquid lens element has 29 structures so that using four liquid lens elements has $29^{4}$ combination structures. Only two of $29^{4}$ combination structures were selected for the EFLs $5 \mathrm{~mm}$ and $7 \mathrm{~mm}$.

The GA optimization found that the better structure for EFL $5 \mathrm{~mm}$ was the liquid lens elements with voltages 41,37 , 60 , and 59 for four liquid elements, respectively. When the voltages were tuned to 57 volts, 51 volts, 32 volts, and 37 volts for four liquid lens elements, a better result was obtained for EFL $7 \mathrm{~mm}$ structure. Table 4 lists the corresponding factors after the GA optimization. Although the GA optimization is a global optimization algorithm, it is very efficient for using damped leased squares to optimize the aspherical coefficient. Therefore, we use the commercial software to find the proper aspherical coefficient after the GA optimization. The aspherical surface is used in the surface 26. Table 5 shows its parameters. Except for the thicknesses of two liquids and the surface curvature between two kinds of liquids, all the thicknesses and curvatures of the proposed zoom lens were constant. The thickness of the two liquids is also constant, which results in the thickness of overall length for the proposed zoom lens being constant. Therefore, a zoom lens applying the liquid lens elements with constant overall length is demonstrated in the proposal by using the GA optimization. Figure 5 shows the lens structures for EFLs $5 \mathrm{~mm}$ and $7 \mathrm{~mm}$. The $F$ numbers are set to 3.2 and 4 for EFLs $5 \mathrm{~mm}$ and $7 \mathrm{~mm}$. The overall length is $24 \mathrm{~mm}$. The 


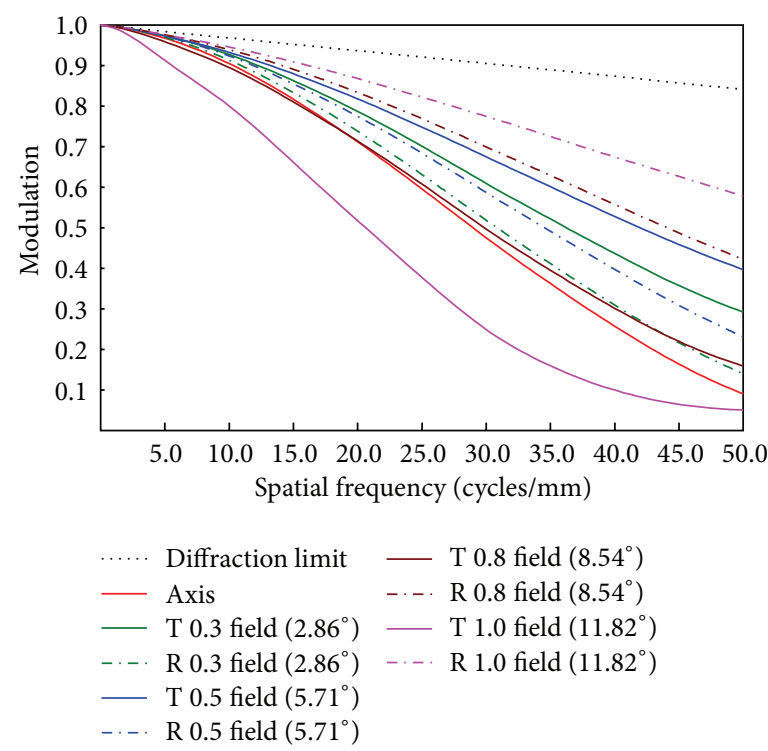

(a)
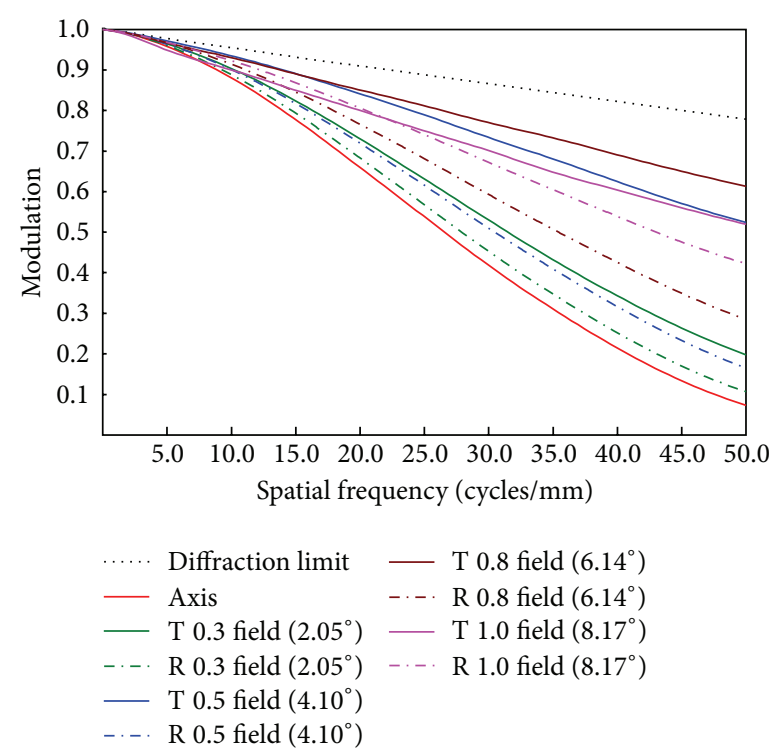

(b)

FIGURE 6: The modulation transformation function: (a) $\mathrm{EFL}=5 \mathrm{~mm}$ and (b) $\mathrm{EFL}=7 \mathrm{~mm}$.

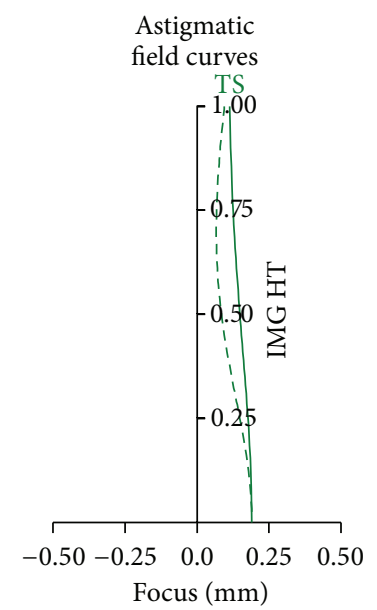

(a)

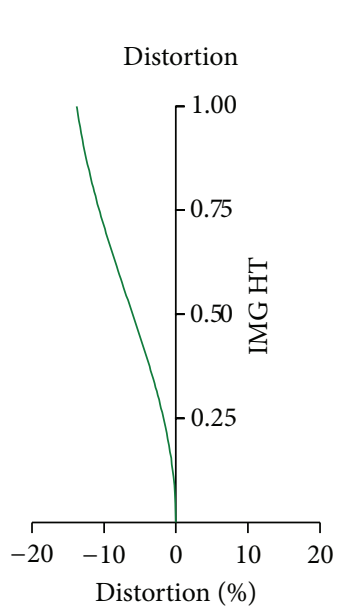

.

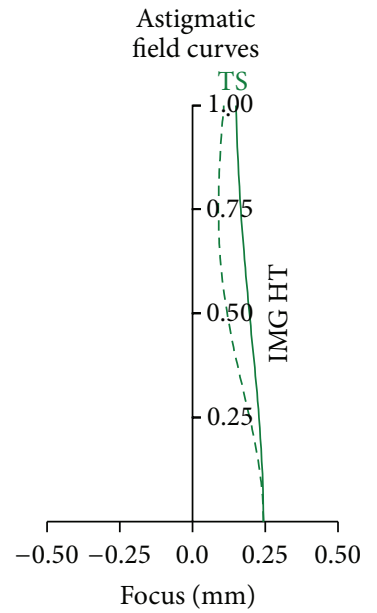

(b)

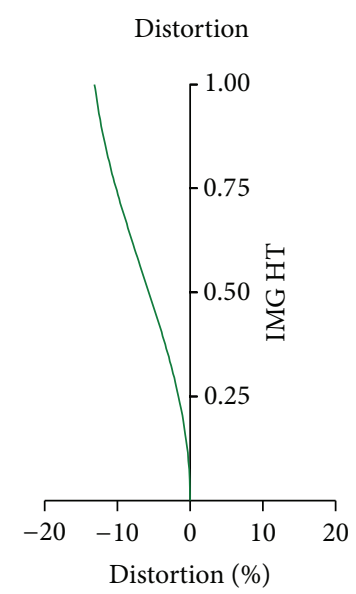

FIGURE 7: Field curvature: (a) EFL $=5 \mathrm{~mm}$ and (b) $\mathrm{EFL}=7 \mathrm{~mm}$.

modulation transfer function (MTF) is shown in Figure 6. Figure 7 shows the filed curvature. Figure 8 shows the spot diagram. It is worth mentioning that the EFL of proposed zoom lens is tuned by liquid elements without moving any lens elements.

When people want to shoot an object with different image size by using a camera with constant EFL, moving the camera close to or far away from the object is the most obvious choice. Moving lens groups is more flexible than using focusvariable elements for implementing zoom function. That is why the conventional zoom lens is realized by moving the lens groups. In [9], the focus and compensating processes can receive the assistance from moving the lens group. The difficulty of the proposal is that the zoom function is only implemented by four liquid elements. Not any lens groups are
TABLE 5: The parameters of surface 26.

\begin{tabular}{lc}
\hline Parameter & Value \\
\hline$Y$ radius & 1.6212 \\
Conic constant & 0.0000 \\
4th-order coefficient & -0.2709 \\
6th-order coefficient & 0.1836 \\
8th-order coefficient & -0.0548 \\
\hline
\end{tabular}

moved to assist zoom function implementation. Moreover, the lens design must satisfy one of 29 discrete statuses of liquid element. As the curvature changed, the thicknesses of both liquids must also be modified to make the optimization 


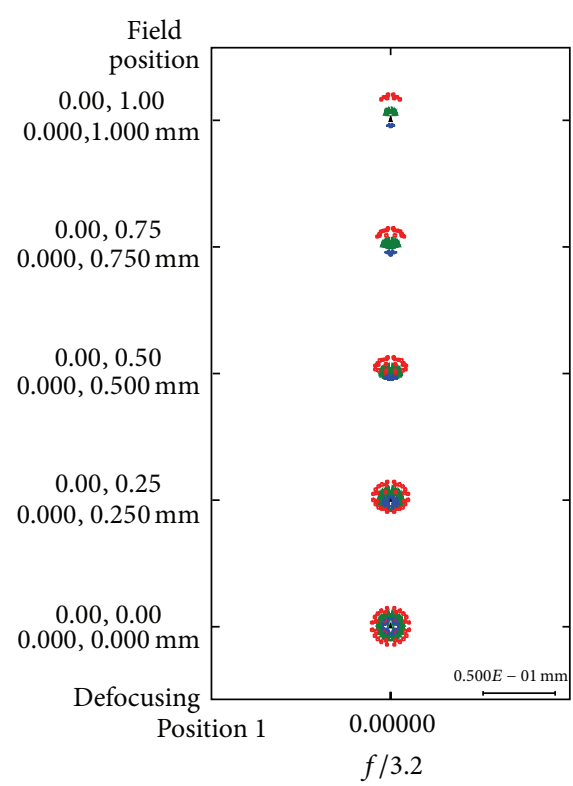

(a)

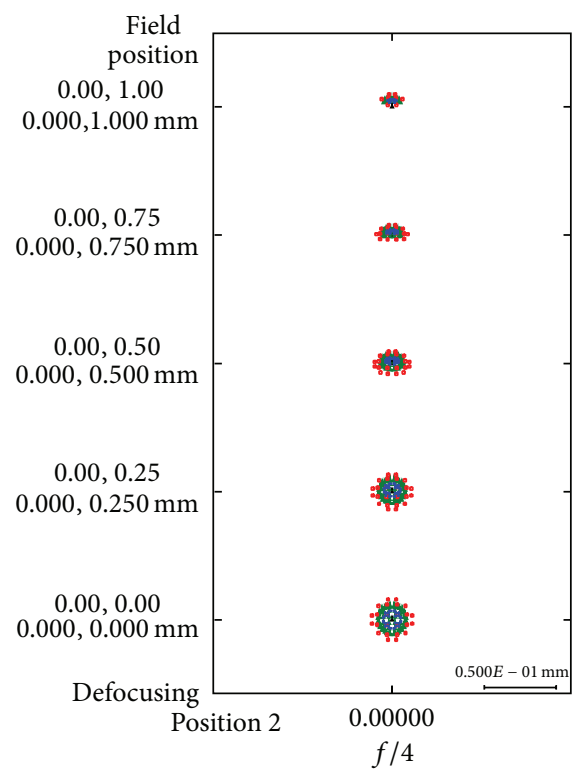

(b)

Figure 8: The spot diagram (a) EFL $=5 \mathrm{~mm}$ and (b) $\mathrm{EFL}=7 \mathrm{~mm}$.

more difficult for commercial software. The other issue is the optical power limited by liquid lens elements because their refractive indices difference between two liquids is small, which results in inability to vary the entire EFL too much. These issues result a zoom lens with liquid lens element is hard to implement under no lens groups moving. Therefore, the field of view of the zoom lens is lower than that of [9]. And the system needs an aspheric surface to enhance image quality. Writing the GA in macro provides a flexible way to search the status of liquid element to make the optimization for zoom lens without any lens groups moving. Adding additional lens elements and liquid lens elements is also one of solutions to promote the zoom ratio. However, the better way is to replace the liquid lens element by another focusvariable element with high optical power. The future work will look for the focus-variable element with high optical power to not only improve the zoom ratio but also reduce using the additional lens elements to shorten the overall length through developing the GA optimization.

\section{Conclusions}

This paper is to apply the liquid lenses to design a zoom lens used in mobile devices. There are 29 discrete curvature variation statuses for liquid lens element, which result in that the common commercial software cannot offer an optimization on the liquid lens element. An optimization called GA is applied to the proposal to search the lens parameters. The proposal employed four liquid elements to design a lens with 1.4 zoom ratio and $24 \mathrm{~mm}$ overall length. Under the $F$ numbers 3.2 and 4.0, a zoom lens with liquid lens elements is designed for EFLs $5 \mathrm{~mm}$ and $7 \mathrm{~mm}$ without moving any lens element. Although it seems difficult to be used in usual mobile devices, the proposal is still to completely achieve a zoom lens design without moving any lens groups, which is very important for mobile devices. Since the zoom ratio and overall length are related to the optical power, the optical power of liquid element used is so small that additional liquid elements need to be put together to increase the optical power for enhancing focus and compensation. When the liquid element with high optical power is developed, the proposal can only employ two liquid elements to achieve the lens with high zoom ratio, which would also shorten the overall length of the lens to meet the requirement for mobile devices.

\section{Conflict of Interests}

The author declares that there is no conflict of interests regarding the publication of this paper.

\section{References}

[1] M. Ye, B. Wang, and S. Sato, "Liquid-crystal lens with a focal length that is variable in a wide range," Applied Optics, vol. 43, no. 35, pp. 6407-6412, 2004.

[2] M. Ye, B. Wang, M. Uchida, S. Yanase, S. Takahashi, and S. Sato, "Focus tuning by liquid crystal lens in imaging system," Applied Optics, vol. 51, no. 31, pp. 7630-7635, 2012.

[3] P. Liebetraut, P. Waibel, P. H. C. Nguyen, P. Reith, B. Aatz, and H. Zappe, "Optical properties of liquids for fluidic optics," Applied Optics, vol. 52, no. 14, pp. 3203-3215, 2013.

[4] H. Ren and S.-T. Wu, "Variable-focus liquid lens," Optics Express, vol. 15, no. 10, pp. 5931-5936, 2007.

[5] L. Dong, A. K. Agarwal, D. J. Beebe, and H. Jiang, "Adaptive liquid microlenses activated by stimuli-responsive hydrogels," Nature, vol. 442, no. 7102, pp. 551-554, 2006.

[6] H. Ren and S.-T. Wu, "Tunable-focus liquid microlens array using dielectrophoretic effect," Optics Express, vol. 16, no. 4, pp. 2646-2652, 2008. 
[7] B. Berge and J. Peseux, "Variable focal lens controlled by an external voltage: an application of electrowetting," European Physical Journal E, vol. 3, no. 2, pp. 159-163, 2000.

[8] Y.-C. Fang and C.-M. Tsai, "Miniature lens design and optimization with liquid lens element via genetic algorithm," Journal of Optics A: Pure and Applied Optics, vol. 10, no. 7, Article ID 075304, 2008.

[9] Y.-C. Fang, C.-M. Tsai, and C.-L. Chung, "A study of optical design and optimization of zoom optics with liquid lenses through modified genetic algorithm," Optics Express, vol. 19, no. 17, pp. 16291-16302, 2011.

[10] J.-H. Sun, B.-R. Hsueh, Y.-C. Fang, J. MacDonald, and C.-C. Hu, "Optical design and multiobjective optimization of miniature zoom optics with liquid lens element," Applied Optics, vol. 48, no. 9, pp. 1741-1757, 2009.

[11] K. Levenberg, "A method for the solution of certain problems in least squares," Quarterly of Applied Mathematics, vol. 2, pp. 164-168, 1994.

[12] H. Matsui and K. Tanaka, "Determination method of an initial damping factor in the damped-least-squares problem," Applied Optics, vol. 33, no. 13, pp. 2411-2418, 1994.

[13] M. Gen and R. Cheng, Genetic Algorithms and Engineering Design, John Wiley \& Sons, New York, NY, USA, 1997.

[14] Y.-C. Fang, C.-M. Tsai, J. MacDonald, and Y.-C. Pai, "Eliminating chromatic aberration in Gauss-type lens design using a novel genetic algorithm," Applied Optics, vol. 46, no. 13, pp. 2401-2410, 2007.

[15] C.-C. Chen, C.-M. Tsai, and Y. C. Fang, "Optical design of LCOS optical engine and optimization with genetic algorithm," Journal of Display Technology, vol. 5, no. 8, pp. 293-305, 2009.

[16] S.-T. Pan, "CSD-coded genetic algorithm on robustly stable multiplierless IIR filter design," Mathematical Problems in Engineering, vol. 2012, Article ID 560650, 15 pages, 2012.

[17] L. Huo, "Optimal design of liquid dampers for structural vibration control based on GA and $H_{\infty}$ Norm," Mathematical Problems in Engineering, vol. 2013, Article ID 191279, 10 pages, 2013.

[18] Y. Q. Ma and H. X. Qiu, "Integrated multiobjective optimal design for active control system based on genetic algorithm," Mathematical Problems in Engineering, vol. 2014, Article ID 748237, 9 pages, 2014.

[19] http://www.varioptic.com/. 

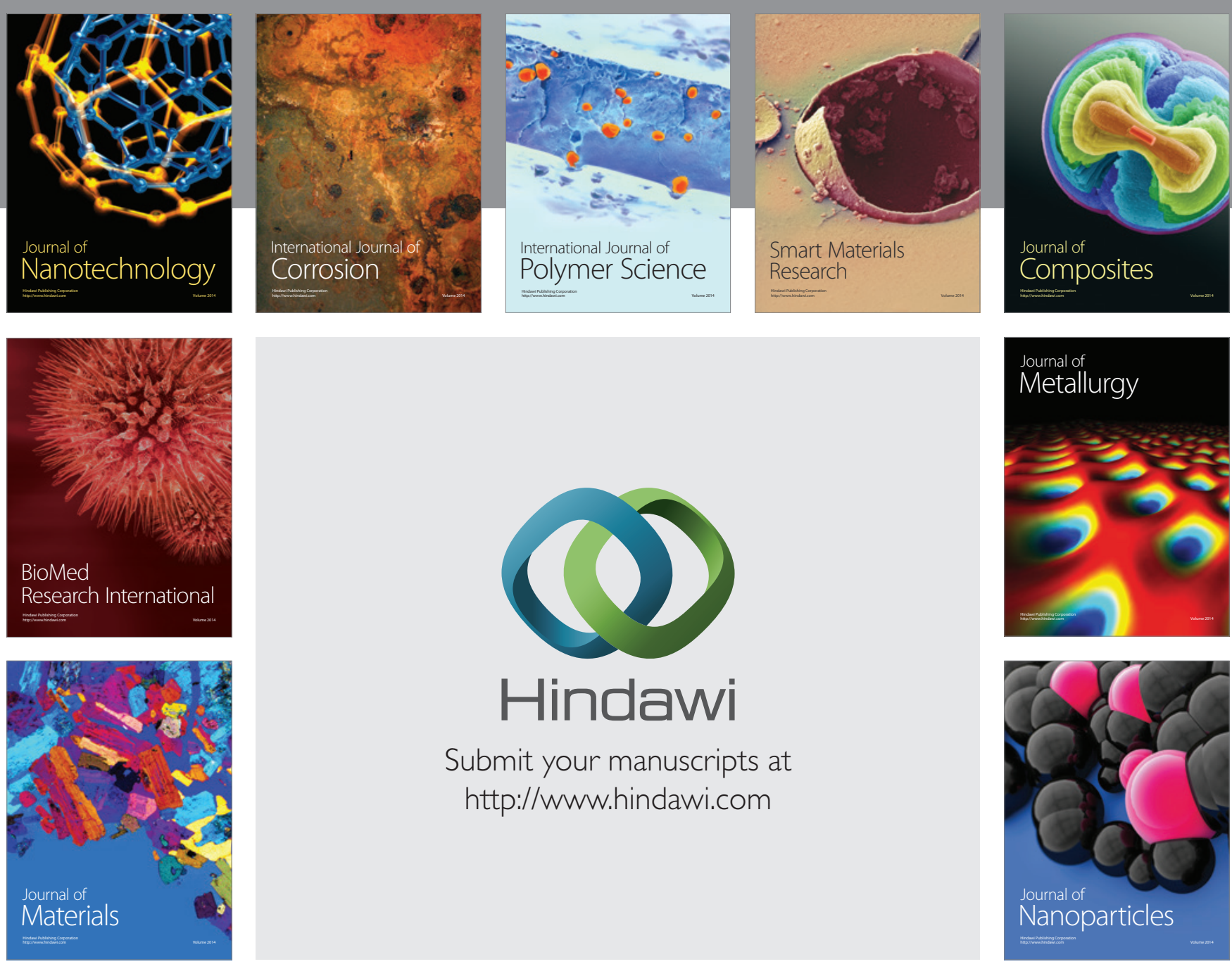

Submit your manuscripts at http://www.hindawi.com
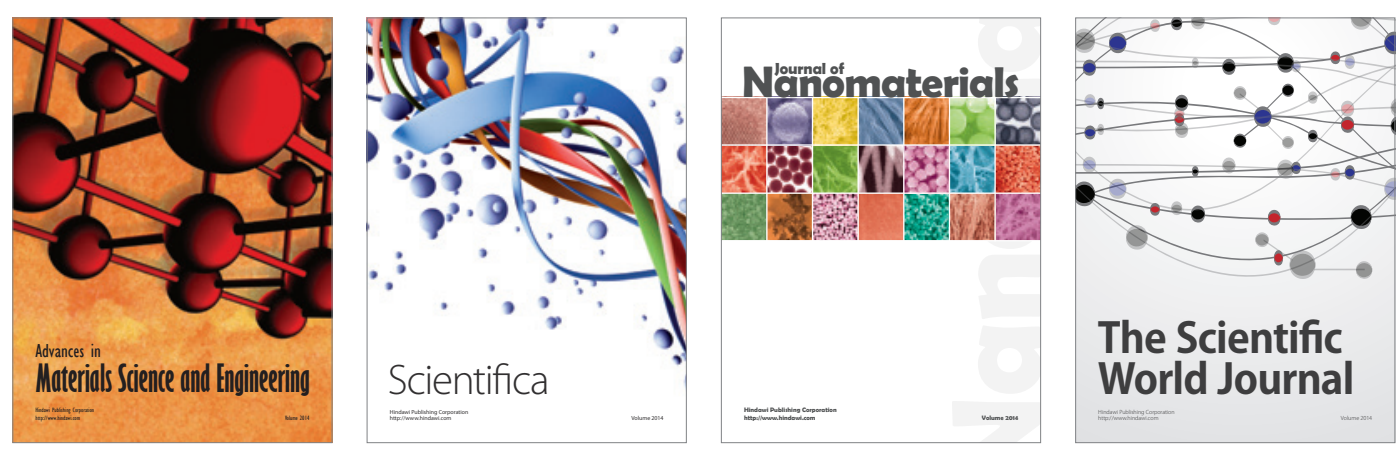

\section{The Scientific World Journal}
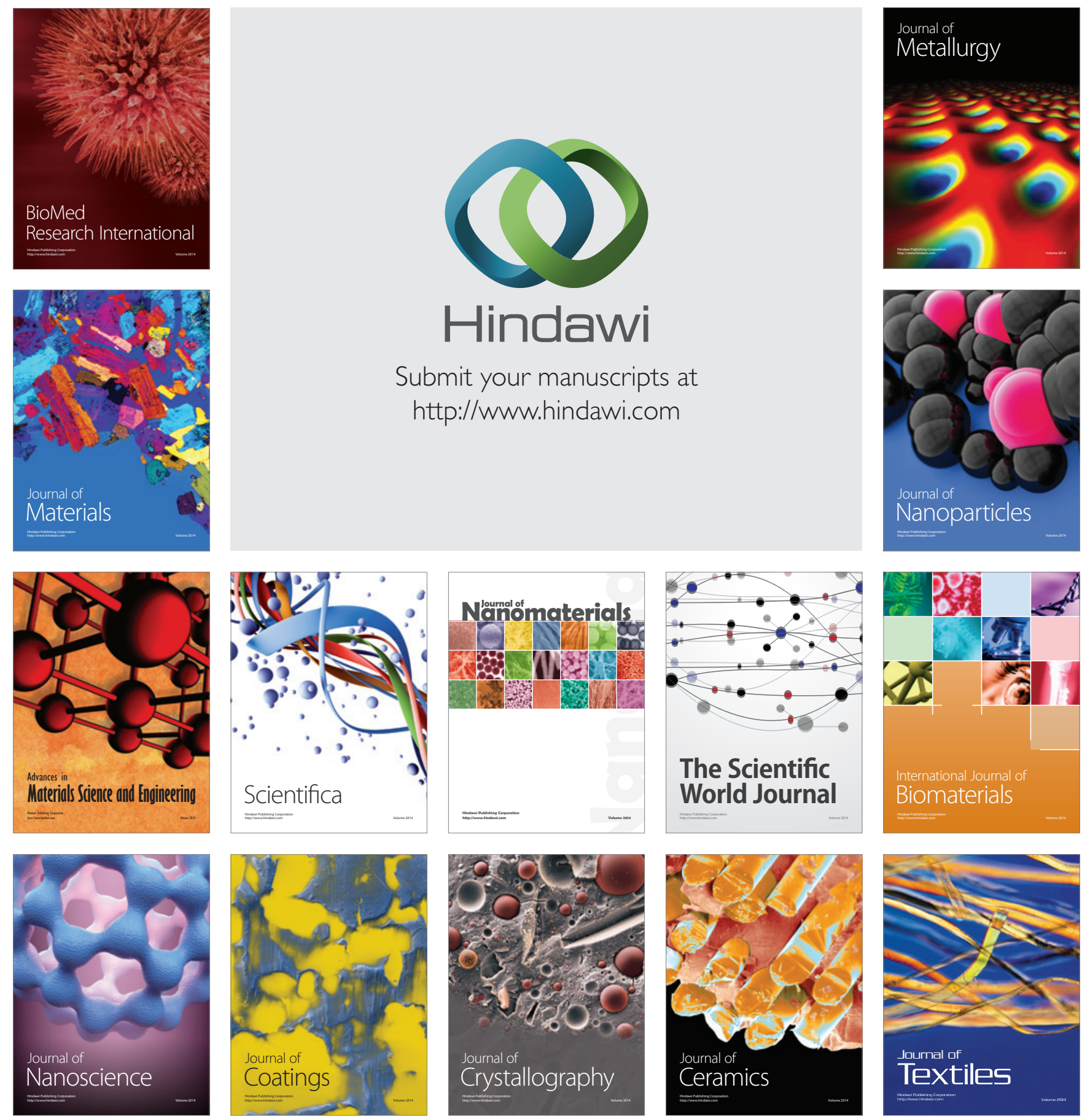